\title{
Paraoxonase 1 activities and genetic variation in childhood obesity
}

\author{
A. I. Rupérez ${ }^{1}$, O. López-Guarnido ${ }^{2}$, F. Gil ${ }^{2}$, J. Olza $^{1}$, M. Gil-Campos ${ }^{3}$, R. Leis ${ }^{4}$, R. Tojo ${ }^{4}$, \\ R. Cañete ${ }^{3}$, A. Gil ${ }^{1}$ and C. M. Aguilera ${ }^{1 *}$ \\ ${ }^{1}$ Department of Biochemistry and Molecular Biology II, Institute of Nutrition and Food Technology, \\ Centre of Biomedical Research, Laboratory 123, University of Granada, Avenida del Conocimiento s $n$, \\ 18100 Armilla, Granada, Spain \\ ${ }^{2}$ Department of Legal Medicine, Toxicology and Anthropology, University of Granada, 18071 Granada, Spain \\ ${ }^{3}$ Unit of Paediatric Endocrinology, Reina Sofia University Hospital, 14004 Córdoba, Spain \\ ${ }^{4}$ Unit of Investigation in Nutrition, Growth and Human Development of Galicia, Paediatric Department, \\ Clinic University Hospital of Santiago, University of Santiago de Compostela, 15706 Santiago de Compostela, Spain
}

(Submitted 19 December 2012 - Final revision received 16 April 2013 - Accepted 18 May 2013 - First published online 21 June 2013)

\section{Abstract}

Changes in paraoxonase 1 (PON1) activities have been observed in a variety of diseases involving oxidative stress, such as CVD. However, its role in obesity has not been fully established. In the present study, we aimed (1) to genotype sixteen PON1 SNP, (2) to measure serum PON1 activities and (3) to correlate these findings with the incidence of childhood obesity and related traits. We conducted a case-control study of 189 normal-weight and 179 obese prepubertal children, and we measured four different PON1 activities: lactonase; paraoxonase; arylesterase; diazoxonase. Although none of these activities was significantly different between the obese and normal-weight children, lactonase activity was found to be positively correlated with HDL-cholesterol and ApoA1 levels and negatively correlated with myeloperoxidase and fatty acid-binding protein 4 levels. Among the sixteen genotyped PON1 SNP, only the intronic SNP rs854566 exhibited a significant association with obesity (OR $0.61,95 \%$ CI $0.41,0.91 ; P=0.016)$. This genetic variant was also associated with increased diazoxonase, lactonase and arylesterase activities and decreased paraoxonase activity. Other genetic variants exhibited different association patterns with serum activities based on their location within the PON1 gene, and SNP that were located within the promoter were strongly associated with lactonase, arylesterase and diazoxonase activities. The functional variant Q192R exhibited the greatest effect on paraoxonase activity $\left(P=5.88 \times 10^{-42}\right)$. In conclusion, SNP rs854566 was negatively associated with childhood obesity and with increased serum PON1 activities in prepubertal children. We determined that lactonase is a reliable indicator of PON1 activities and should be included in future studies of PON1 function.

Key words: Paraoxonase 1 gene: Genetic polymorphisms: Obesity: Children

In humans, paraoxonase 1 (PON1) is primarily expressed in the liver and can be detected circulating in the plasma in association with $\mathrm{HDL}^{(1)}$. PON1 is involved in the metabolism of lipoprotein phospholipids and inhibits the lipid peroxidation of LDL and $\mathrm{HDL}^{(1,2)}$. Changes in PON1 activities have been shown to lead to a variety of diseases that involve oxidative stress, including CVD, Alzheimer's disease, chronic renal failure, the metabolic syndrome and chronic liver impairment ${ }^{(3)}$.

With the prevalence of obesity and metabolic disorders, such as type 2 diabetes, being on the rise, these conditions will probably remain the major contributors to cardiovascular mortality and morbidity in the twenty-first century. A growing body of evidence that suggests that obesity-induced oxidative stress plays an important role in adults and children has emerged in the past few years. Recently, our group has demonstrated elevated levels of plasma myeloperoxidase (MPO) in prepubertal obese children, and we detected that the expression of this enzyme was correlated with biomarkers for inflammation and cardiovascular risk, such as high-sensitivity C-reactive protein, matrix metalloproteinase-9 and resistin ${ }^{(4)}$. Taken together, these findings highlight the need to investigate the molecular mechanisms linking metabolic stress, obesity and, ultimately, CVD. Furthermore, the finding that PON1 is expressed in the interstitial space of adipose tissue ${ }^{(5)}$ suggests a role for the PON1 enzyme in these disorders.

Relatively little work has been conducted to address the role of PON1 in obesity, and the small number of studies that have been carried out are not always in agreement. For example,

Abbreviations: HDL-C, HDL-cholesterol; LD, linkage disequilibrium; MPO, myeloperoxidase; Ox-LDL, oxidised LDL; PON1, paraoxonase 1.

*Corresponding author: C. M. Aguilera, fax +34958 248960, email caguiler@ugr.es 
decreased levels of paraoxonase and arylesterase activities have been observed in obese adults ${ }^{(6,7)}$ and children ${ }^{(8)}$, although another study has observed no changes in paraoxonase activity in adults with the metabolic syndrome or obesity $^{(9)}$. However, the lack of a strong link between PON1 activities and oxidative stress could be due to differences in measurement techniques and data analysis between these studies. For example, the actual physiological substrates of PON1 are unclear, and the available methods for testing PON1 activities use a variety of synthetic substrates, including paraoxon (paraoxonase activity), phenylacetate (arylesterase activity) and diazoxon (diazoxonase activity). Although less commonly reported in the literature, researchers have also tested PON1 lactonase activity using a variety of lactones, such as dihydrocoumarin. This activity has been proposed to be responsible for oxidised-lipid hydrolysis, HDL-mediated macrophage cholesterol efflux $^{(10)}$ and homocysteinethiolactone hydrolysis ${ }^{(11)}$. Due to these technical issues, measurements of PON1 activities will probably differ depending on the specific substrate used ${ }^{(12,13)}$. Another issue is that serum PON1 activities are strongly affected by enzymatic genotype as well as by exogenous factors, such as age, diet and drug, alcohol and tobacco use ${ }^{(14)}$. Of these factors, genetics are the main determinant of PON1 variability, and PON1 activities can differ by as much as a factor of 40 across a population of healthy individuals from the same ethnic group ${ }^{(15)}$. Although nearly 200 SNP have been described for $P O N 1^{(16)}$, it is common for authors to state only two known coding polymorphisms in this gene: Q192R (rs662) and L55M (rs854560). To date, no strong links have been observed between the presence of these SNP and obesity, and although a number of association studies have been performed $^{(17-19)}$, the findings of these studies are inconsistent. With this in mind, we carried out a comprehensive association study in obese prepubertal children that had additional PON1 SNP as well as the previously characterised missense variations. In addition, we measured serum PON1 activities using four different substrates (dihydrocoumarin, paraoxon, phenylacetate and diazoxon) and correlated these results with a wide variety of plasma biomarkers, including markers for oxidative stress, insulin resistance, obesity and cardiovascular risk, to determine their association with PON1 activities and genetic variability.

\section{Experimental methods}

\section{Study population}

In the present case-control study, we recruited 179 obese children (95 males and 84 females) and 189 normal-weight children (109 males and 80 females), aged 4-13 years, from two Spanish cities (Córdoba and Santiago de Compostela). Childhood obesity was defined according to Cole et al. ${ }^{(20)}$. To be included in the study, it was required that the children were prepubertal and were not suffering from nutritional diseases or endogenous obesity. Children who were suffering from disease or malnutrition, currently in puberty or using medications to alter blood pressure, glucose levels or lipid metabolism were excluded from the study. Following an initial assessment at school or a primary-care centre, children who met the inclusion criteria were invited for a clinical examination at a participating hospital. The parents or guardians of the children were informed concerning the purpose and procedures of the study before written consent was obtained; all of the children agreed to participate in the study. Sex hormone levels were measured to confirm that the children were prepubertal (data not shown). The protocol was performed in accordance with the Declaration of Helsinki (Edinburgh 2000, revised), the recommendations of the Good Clinical Practice of the CEE (Document 111/3976/88, July, 1990) and the current Spanish regulations dictating clinical investigations in human subjects (RD 223/04 on Clinical Assays). The present study was approved by the Ethics Committee on Human Research of the University of Granada, the Ethics Committee of the Reina Sofía University Hospital of Córdoba and the Bioethics Committee of the University of Santiago de Compostela.

\section{Anthropometric and biochemical measurements}

Anthropometric measurements were performed by a single examiner with the children in bare feet and dressed in their underwear. Body weight $(\mathrm{kg})$, height $(\mathrm{cm})$ and waist circumference $(\mathrm{cm})$ were measured using standardised procedures and were used to calculate the BMI of the children. Obesity was defined according to BMI, using the age- and sex-specific cut-off points proposed by Cole et al $^{(20)}$ (linked to adult cutoffs of 25 and $30 \mathrm{~kg} / \mathrm{m}^{2}$ ). Blood pressure was measured three times for each individual by the same examiner, according to international recommendations. Blood samples were drawn from the antecubital vein after the children had fasted overnight. Biochemical analyses and measurements of specific biomarkers, including adiponectin, leptin, oxidised LDL (ox-LDL) and high-sensitivity C-reactive protein, were performed as described previously ${ }^{(21)}$. The levels of MPO, matrix metalloproteinase- 9 and both the total and active forms of plasminogen activator inhibitor-1 were measured as described previously ${ }^{(4)}$. Plasma total antioxidant capacity was assessed using the spectrophotometric antioxidant assay kit from Cayman (catalogue no. 709001). Fatty acid-binding protein 4 concentrations were measured using ELISA (catalogue no. RD191036200R; BioVendor). Quantitative insulin sensitivity check index and homeostasis model assessment for insulin resistance scores were calculated using plasma glucose and insulin levels. Retinol, $\alpha$-tocopherol and $\beta$-carotene levels were analysed using HPLC, as described previously ${ }^{(22)}$.

\section{DNA isolation and genotyping}

Genomic DNA was extracted using the QIAamp Blood kit (Qiagen). Based on their location with respect to the PON1 gene, a total of sixteen SNP were selected from the HapMap and NCBI databases. First, each missense variation was selected. Then, SNP were selected from the promoter $3^{\prime}$ untranslated region (UTR) and $5^{\prime}$ UTR. All SNP had minor allele frequencies greater than 0.05 in the Caucasian 
population. Table S1 (available online) describes the main characteristics of the SNP included in the present study.

Genotyping was performed using the Illumina GoldenGate Assay (Illumina), as described previously ${ }^{(21)}$. The success rates for genotyping were $>95 \%$ for nearly all of the SNP, with the exception of rs705379, which was excluded from further analysis. The Hardy-Weinberg equilibrium for each SNP was determined with the exact test using the PLINK software program (version 1.07; http://pngu.mgh.harvard.edu/ purcell/ plink) ${ }^{(23)}$. The Hardy-Weinberg equilibrium $P$ values were greater than 0.05 in both the obese and normal-weight groups for all the SNP, and the observed allele frequencies of the SNP in the present study were similar to those reported in the HapMap database for Caucasians (Table S1, available online). Linkage disequilibrium (LD) was analysed using the Haploview 4.2 software program (http://www.broad.mit. edu/mpg/haploview/) ${ }^{(24)}$

\section{Determination of paraoxonase 1 activities}

Serum PON1 activities were determined using four different substrates (dihydrocoumarin, paraoxon, phenylacetate and diazoxon), as described previously ${ }^{(15,25)}$. Arylesterase, diazoxonase and lactonase enzyme assays were performed using a Lambda-2 spectrophotometer (Perkin Elmer Inc.). PON1 paraoxonase activity was determined using a multidetection microplate reader (BioTek Synergy HT; BioTek Instruments Inc.). The samples were assayed in duplicate, and the average value was used for further analysis. The non-enzymatic hydrolysis rate for each substrate was measured by substituting serum for buffer, and this value was subtracted from the total rate of hydrolysis.

\section{Statistical analyses}

All of the statistical analyses were performed using either the PLINK or SPSS software program (version 15.0.1; SPSS, Inc.). The normal distribution of the clinical parameter data was assessed using the Kolmogorov-Smirnov test. Normally distributed continuous variables are expressed as means with their standard errors, and non-normally distributed variables are expressed as medians and ranges. The values of glucose, TAG, leptin, $\alpha$-tocopherol, $\beta$-carotene and ox-LDL were logarithmically transformed to approximate normal distributions; the values of insulin, homeostasis model assessment for insulin resistance and adiponectin were transformed using the squareroot transformation technique, and the quantitative insulin sensitivity check index was inversely transformed. Mean comparisons between continuous variables for the obese and normal-weight children were performed using Student's $t$ test for unpaired samples. The paraoxonase activity could not be transformed to a normal distribution due to its bimodal nature; therefore, the Mann-Whitney $U$ test was applied.

Genotypic relative risk was assessed by comparing the obese group with the normal-weight group and by calculating the OR and the 95\% CI using a logistic regression analysis under an additive model adjusted for age and sex. A meta-analysis was performed to discard population stratification due to the two cities the children were recruited from.

\section{Results}

\section{Patient characteristics}

Table 1 summarises the demographic, clinical and biochemical characteristics of the study population. As expected, the obese children had significantly greater values for weight, BMI, BMI $z$-score, waist circumference, leptin and fatty acid-binding protein 4 and significantly lower adiponectin levels. Metabolic syndrome parameters, such as systolic and diastolic blood pressure, TAG, insulin and homeostasis model assessment for insulin resistance values, were significantly higher in the obese children, whereas the quantitative insulin sensitivity check index, HDL-cholesterol (HDL-C) and ApoA1 values were lower in this group. The levels of the cardiovascular risk biomarkers MPO, active plasminogen activator inhibitor1 and total plasminogen activator inhibitor- 1 were significantly increased in the obese children, whereas no difference was observed for that of matrix metalloproteinase-9 compared with that in the normal-weight group. The levels of the oxidative stress biomarkers ox-LDL, retinol and $\alpha$-tocopherol were not significantly different between the groups, whereas those of $\beta$-carotene and total antioxidant capacity were lower in the obese children. Inflammation was also detected in the obese group, as indicated by greater high-sensitivity C-reactive protein values.

\section{Paraoxonase 1 activities in childhood obesity and their correlations with metabolic, cardiovascular risk and oxidative stress biomarkers}

None of the PON1 activities differed between the obese and normal-weight children (Table S2, available online).

Lactonase activity showed significant correlations with the levels of the greatest number of analysed biomarkers compared with the other PON1 activities (Table 2). Lactonase activity was positively correlated with the levels of several biomarkers involved in lipid metabolism, including total cholesterol, LDL-cholesterol, HDL-C and ApoA1. Moreover, lactonase activity was positively correlated with the levels of several antioxidant molecules, including retinol and $\alpha$-tocopherol, and it was negatively correlated with those of fatty acid-binding protein 4 and MPO, which have recently been described as biomarkers for CVD risk.

With respect to the activities of the other enzymes, total cholesterol, HDL-C and ApoA1 levels were correlated with diazoxonase $(r$ 0.174, $P<0.001 ; r 0.247, P<0.001 ; r 0.121$, $P=0.022$, respectively $)$ and arylesterase $(r 0.232, P<0.001$; $r$ 0.222, $P<0.001 ; r 0.225, P<0.001$, respectively) activities. In addition, retinol levels were positively correlated with lactonase and arylesterase $(r 0 \cdot 130, P=0 \cdot 014)$ activities. However, paraoxonase activity was not significantly correlated with any of the tested biomarker levels or anthropometric measurements; similar results were observed in both the experimental and control groups (data not shown). No significant correlations were observed between any of the PON1 activities and weight, BMI, BMI $z$-score, waist circumference, DBP, glucose, insulin, quantitative insulin sensitivity check index, homeostasis model assessment for insulin resistance, TAG, 
Table 1. Demographic, clinical and biochemical characteristics of the children included in the present study

(Mean values with their standard errors)

\begin{tabular}{|c|c|c|c|c|c|}
\hline \multirow[b]{2}{*}{ Variables } & \multicolumn{2}{|c|}{ Normal weight } & \multicolumn{2}{|c|}{ Obese } & \multirow[b]{2}{*}{$P^{*}$} \\
\hline & Mean & SEM & Mean & SEM & \\
\hline \multicolumn{6}{|l|}{$\operatorname{Sex}(n)$} \\
\hline Male & \multirow{2}{*}{\multicolumn{2}{|c|}{$\begin{array}{c}109 \\
80\end{array}$}} & \multicolumn{2}{|c|}{95} & \\
\hline Female & & & & & \\
\hline Age (years) & 8.9 & 0.1 & $8 \cdot 6$ & $0 \cdot 1$ & 0.106 \\
\hline Weight (kg) & $29 \cdot 1$ & 0.4 & $51 \cdot 0$ & 1.0 & $<0.001$ \\
\hline BMI $\left(\mathrm{kg} / \mathrm{m}^{2}\right)$ & $16 \cdot 61$ & 0.11 & $26 \cdot 85$ & 0.28 & $<0.001$ \\
\hline BMI $z$-score & -0.19 & 0.04 & 3.52 & 0.11 & $<0.001$ \\
\hline WC (cm) & $58 \cdot 2$ & 0.5 & $81 \cdot 0$ & 1.0 & $<0.001$ \\
\hline $\mathrm{SBP}(\mathrm{mmHg})$ & 96 & 1 & 110 & 1 & $<0.001$ \\
\hline $\mathrm{DBP}(\mathrm{mmHg})$ & 58 & 1 & 69 & 1 & $<0.001$ \\
\hline Glucose (mg/l) & 830 & 10 & 840 & 10 & 0.778 \\
\hline Insulin (pmol/l) & 35.88 & 1.38 & 71.76 & 3.45 & $<0.001$ \\
\hline QUICKI & 0.390 & 0.003 & 0.351 & 0.002 & $<0.001$ \\
\hline HOMA-IR & 1.08 & 0.04 & $2 \cdot 17$ & 0.11 & $<0.001$ \\
\hline Total cholesterol (mg/l) & 1720 & 20 & 1660 & 20 & 0.052 \\
\hline TAG (mg/l) & 540 & 10 & 760 & 30 & $<0.001$ \\
\hline HDL-C (mg/l) & 650 & 10 & 520 & 10 & $<0.001$ \\
\hline LDL-C (mg/l) & 940 & 20 & 970 & 20 & 0.227 \\
\hline ApoA1 (mg/l) & 1510 & 20 & 1330 & 20 & $<0.001$ \\
\hline ApoB $(\mathrm{mg} / \mathrm{l})$ & 670 & 10 & 720 & 10 & 0.005 \\
\hline FABP-4 (ng/ml) & 9.54 & 0.60 & $27 \cdot 23$ & 1.47 & $<0.001$ \\
\hline Adiponectin (mg/l) & $27 \cdot 81$ & 0.81 & 22.57 & 0.84 & $<0.001$ \\
\hline Leptin $(\mu \mathrm{g} / \mathrm{l})$ & $4 \cdot 15$ & 0.29 & $22 \cdot 70$ & 1.03 & $<0.001$ \\
\hline ox-LDL (mg/l) & 2.057 & 0.213 & 2.045 & 0.191 & 0.502 \\
\hline Retinol $(\mu \mathrm{g} / \mathrm{ml})$ & 0.247 & 0.005 & 0.254 & 0.005 & 0.351 \\
\hline$\alpha$-Tocopherol $(\mu \mathrm{g} / \mathrm{ml})$ & 7.965 & 0.129 & 7.907 & 0.160 & 0.776 \\
\hline$\beta$-Carotene $(\mu \mathrm{g} / \mathrm{ml})$ & 0.148 & 0.009 & 0.086 & 0.004 & $<0.001$ \\
\hline TAC (mM-Trolox) & $2 \cdot 18$ & 0.05 & 1.79 & 0.07 & $<0.001$ \\
\hline hsCRP (mg/l) & 0.986 & 0.285 & 3.520 & 0.286 & $<0.001$ \\
\hline MMP-9 ( $\mu \mathrm{g} / \mathrm{l})$ & $83 \cdot 21$ & $3 \cdot 72$ & 94.40 & $5 \cdot 58$ & 0.815 \\
\hline MPO $(\mu \mathrm{g} / \mathrm{l})$ & $13 \cdot 84$ & 1.43 & 24.63 & $2 \cdot 60$ & $<0.001$ \\
\hline Active PAl-1 ( $\mu \mathrm{g} / \mathrm{l})$ & 4.73 & 0.26 & $12 \cdot 70$ & 0.78 & $<0.001$ \\
\hline Total PAI-1 $(\mu \mathrm{g} / \mathrm{l})$ & 18.54 & 0.95 & 29.84 & 1.56 & $<0.001$ \\
\hline
\end{tabular}

WC, waist circumference; SBP, systolic blood pressure; DBP, diastolic blood pressure; QUICKI, quantitative insulin sensitivity check index; HOMA-IR, homeostasis model assessment for insulin resistance; HDL-C, HDL-cholesterol; LDL-C, LDL-cholesterol; FABP-4, fatty acid-binding protein 4; ox-LDL, oxidised LDL; TAC, total antioxidant capacity; Trolox, 6-hydroxy-2,5,7,8-tetramethylchroman-2-carboxylic acid; hsCRP, high-sensitivity C-reactive protein; MMP-9, matrix metalloproteinase-9; MPO, myeloperoxidase; PAI-1, plasminogen activator inhibitor-1.

* Student's $t$ test.

$\alpha$-tocopherol, $\beta$-carotene, total antioxidant capacity, ox-LDL, high-sensitivity C-reactive protein, adiponectin or leptin values (data not shown).

We also examined the relationship between the different enzymatic activities within our cohort. Lactonase activity was closely associated with both arylesterase $(r 0.895, P<0 \cdot 001)$ and diazoxonase $(r 0.673, P<0.001)$ activities. Arylesterase activity was also associated with diazoxonase activity ( $r$ 0.787, $P<0.001)$. However, paraoxonase activity was only weakly correlated with lactonase activity $(r 0 \cdot 172, P=0 \cdot 001)$.

\section{Association of paraoxonase 1 SNP with the incidence of childhood obesity and related traits}

The results of the logistic regression analysis and the SNP allele frequencies are given in Table 3. The only SNP that showed an association with obesity was rs854566, which showed a negative association (OR 0.61, 95\% CI 0.41, 0.91; $P=0.016)$ under the additive model of inheritance. This associ- ation was not significant after Bonferroni correction for multiple testing (data not shown). Other models of inheritance such as recessive model did not add strength to this association. Population stratification was discarded by conducting a metaanalysis using the individual association results from each of the examined populations (Córdoba and Santiago de Compostela) $(Q=0 \cdot 906, I=0 \cdot 00)$. The missense SNP Q192R and L55M were distributed equally among the study group populations, and they did not show any association with obesity.

Phenotypic associations between the Q192R, L55M and rs854566 SNP and the anthropometric measurements as well as the biomarkers for metabolic, oxidative stress and cardiovascular risk are given in Table S3 (available online). The associations between SNP rs854566 and BMI, BMI $z$-score and weight were significant $(P<0.05)$, although these associations lost their significance when the samples were divided into obese and normal-weight groups (data not shown). None of the oxidative stress biomarkers (ox-LDL, total antioxidant capacity, retinol, $\alpha$-tocopherol and $\beta$-carotene) was 
Table 2. Correlations between serum paraoxonase 1 lactonase activity and metabolic, cardiovascular risk and oxidative stress biomarkers

\begin{tabular}{|c|c|c|}
\hline Biomarkers & $r$ & $P$ \\
\hline Weight (kg) & -0.057 & 0.274 \\
\hline $\operatorname{BMI}\left(\mathrm{kg} / \mathrm{m}^{2}\right)$ & -0.062 & 0.240 \\
\hline BMI $z$-score & -0.073 & 0.162 \\
\hline WC $(\mathrm{cm})$ & 0.005 & 0.923 \\
\hline $\mathrm{SBP}(\mathrm{mmHg})$ & -0.075 & 0.167 \\
\hline $\mathrm{DBP}(\mathrm{mmHg})$ & -0.077 & 0.154 \\
\hline Glucose (mg/l) & 0.034 & 0.525 \\
\hline Insulin (pmol/l) & -0.075 & 0.160 \\
\hline QUICKI & -0.056 & 0.300 \\
\hline HOMA-IR & -0.073 & 0.174 \\
\hline Total cholesterol $(\mathrm{mg} / \mathrm{l})$ & 0.248 & $<0.001$ \\
\hline TAG $(\mathrm{mg} / \mathrm{l})$ & -0.037 & 0.480 \\
\hline HDL-C (mg/l) & 0.235 & $<0.001$ \\
\hline LDL-C (mg/l) & 0.131 & 0.013 \\
\hline ApoA1 (mg/l) & 0.254 & $<0.001$ \\
\hline ApoB $(\mathrm{mg} / \mathrm{l})$ & 0.078 & 0.138 \\
\hline FABP-4 & -0.227 & 0.033 \\
\hline Adiponectin (mg/l) & 0.079 & 0.137 \\
\hline Leptin $(\mu \mathrm{g} / \mathrm{l})$ & -0.024 & 0.650 \\
\hline ox-LDL (mg/l) & -0.043 & 0.410 \\
\hline Retinol $(\mu \mathrm{g} / \mathrm{ml})$ & 0.259 & $<0.001$ \\
\hline$\alpha$-Tocopherol $(\mu \mathrm{g} / \mathrm{ml})$ & 0.167 & 0.001 \\
\hline$\beta$-Carotene $(\mu \mathrm{g} / \mathrm{ml})$ & 0.075 & 0.158 \\
\hline TAC (mM-Trolox) & -0.042 & 0.426 \\
\hline hsCRP (mg/l) & -0.006 & 0.915 \\
\hline MMP-9 $(\mu \mathrm{g} / \mathrm{l})$ & -0.063 & 0.230 \\
\hline MPO $(\mu \mathrm{g} / \mathrm{l})$ & -0.110 & 0.037 \\
\hline Active PAI-1 ( $\mu \mathrm{g} / \mathrm{l})$ & 0.012 & 0.826 \\
\hline Total PAI-1 $(\mu \mathrm{g} / \mathrm{l})$ & 0.005 & 0.923 \\
\hline \multicolumn{3}{|c|}{$\begin{array}{l}\text { WC, waist circumference; SBP, systolic blood pressure; DBP, } \\
\text { diastolic blood pressure; QUICKI, quantitative insulin sensitivity } \\
\text { check index; HOMA-IR, homeostasis model assessment for } \\
\text { insulin resistance; HDL-C, HDL-cholesterol; LDL-C, LDL-choles- } \\
\text { terol; FABP-4, fatty acid-binding protein 4; ox-LDL, oxidised } \\
\text { LDL; TAC, total antioxidant capacity; Trolox, 6-hydroxy-2,5,7,8- } \\
\text { tetramethylchroman-2-carboxylic acid; hsCRP, high-sensitivity } \\
\text { C-reactive protein; MMP-9, matrix metalloproteinase-9; MPO, } \\
\text { myeloperoxidase; PAI-1, plasminogen activator inhibitor-1. }\end{array}$} \\
\hline
\end{tabular}

significantly associated with the examined SNP, which was also true for the insulin resistance and cardiovascular risk biomarkers.

\section{Effects of paraoxonase 1 SNP on paraoxonase 1 activities}

All of the examined SNP showed a certain effect on PON1 activities, with the exception of rs3735590, which is located in the $3^{\prime}$ UTR (Table 4). The remaining SNP showed different association patterns that varied according to their position within the PON1 gene and according to their LD values. The LD analysis (Fig. 1) identified three blocks defined by high $r^{2}$ values between the SNP.

SNP located in the promoter region (rs854571, rs854572, rs854573, rs705382, rs757158 and rs13236941) were strongly associated with lactonase, arylesterase and diazoxonase activities, whereas their associations with paraoxonase activity were much weaker; only SNP rs854572 and rs757158 showed significant associations with paraoxonase activity. Block 3 corresponds to the SNP located in the promoter region.

The intronic SNP rs854566, which was negatively associated with obesity, showed significant effects on all of the tested PON1 activities. The presence of the protective allele (A) was highly associated with increased diazoxonase, lactonase and arylesterase activities and more weakly associated with decreased paraoxonase activity. This SNP was not included in any block due to its weak LD values with neighbouring SNP.

With respect to the missense mutations, rs662 (Q192R) showed the greatest effect on paraoxonase activity, which was highly significant $(\beta=131 \cdot 5,95 \%$ CI $115 \cdot 0, \quad 147 \cdot 9$; $\left.P=5.88 \times 10^{-42}\right)$. This variant was also excluded from the LD blocks due to its weak LD values $\left(r^{2}<0 \cdot 28\right)$ with the other SNP. With respect to rs854560 (L55M), individuals with the 55LL genotype showed greater diazoxonase, lactonase, arylesterase and paraoxonase activities. The intronic SNP rs705378 shared the same associations as rs854560 due to

Table 3. Logistic regression analysis of the PON1 SNP and obesity under the additive model of inheritance (Odds ratios and $95 \%$ confidence intervals)

\begin{tabular}{|c|c|c|c|c|c|c|c|c|c|c|c|c|}
\hline \multirow[b]{2}{*}{ SNP } & \multirow[b]{2}{*}{ Allele 1/allele 2} & \multicolumn{3}{|c|}{ Obese } & \multicolumn{3}{|c|}{ Normal weight } & \multicolumn{2}{|r|}{ MAF } & \multirow[b]{2}{*}{$\mathrm{OR}^{\star}$} & \multirow[b]{2}{*}{$95 \% \mathrm{Cl}$} & \multirow[b]{2}{*}{$P$ (additive model $) \dagger$} \\
\hline & & 11 & 12 & 22 & 11 & 12 & 22 & Obese & Normal weight & & & \\
\hline rs13236941 & $\mathrm{G} / \mathrm{A}$ & 135 & 40 & 2 & 137 & 38 & 5 & 0.124 & 0.133 & 0.95 & $0.62,1.48$ & 0.829 \\
\hline rs757158 & $\mathrm{G} / \mathrm{A}$ & 75 & 79 & 23 & 72 & 80 & 29 & 0.353 & 0.381 & 0.89 & $0.66,1.20$ & 0.451 \\
\hline rs705382 & $\mathrm{G} / \mathrm{C}$ & 86 & 78 & 13 & 88 & 70 & 23 & 0.294 & 0.320 & 0.90 & $0.67,1.24$ & 0.517 \\
\hline rs854573 & $A / G$ & 109 & 63 & 5 & 108 & 63 & 10 & 0.206 & 0.229 & 0.88 & $0.61,1.27$ & 0.498 \\
\hline rs854572 & $\mathrm{G} / \mathrm{C}$ & 70 & 81 & 26 & 68 & 78 & 35 & 0.376 & 0.409 & 0.88 & $0.66,1.18$ & 0.382 \\
\hline rs854571 & $\mathrm{G} / \mathrm{A}$ & 97 & 74 & 6 & 97 & 71 & 13 & 0.243 & 0.268 & 0.87 & $0.62,1 \cdot 24$ & 0.451 \\
\hline rs854566 & $\mathrm{G} / \mathrm{A}$ & 126 & 50 & 1 & 112 & 59 & 10 & 0.147 & 0.218 & 0.62 & $0.41,0.91$ & 0.016 \\
\hline rs705378 & $\mathrm{C} / \mathrm{A}$ & 60 & 90 & 27 & 68 & 83 & 30 & 0.407 & 0.395 & 1.04 & $0.77,1.41$ & 0.796 \\
\hline rs854560 & $\mathrm{A}($ Leu $) / \mathrm{T}$ (Met) & 60 & 90 & 27 & 68 & 83 & 30 & 0.407 & 0.395 & 1.04 & $0.77,1.41$ & 0.796 \\
\hline rs3917527 & $A / G$ & 165 & 12 & 0 & 161 & 20 & 0 & 0.034 & 0.055 & 0.57 & $0.27,1 \cdot 21$ & 0.143 \\
\hline rs662 & $A(G \ln ) / G(A r g)$ & 90 & 72 & 15 & 85 & 79 & 16 & 0.288 & 0.308 & 0.90 & $0 \cdot 65,1 \cdot 24$ & 0.518 \\
\hline rs854552 & $A / G$ & 117 & 53 & 7 & 112 & 57 & 12 & 0.189 & 0.224 & 0.80 & $0.56,1.14$ & 0.217 \\
\hline rs854551 & $\mathrm{G} / \mathrm{A}$ & 132 & 41 & 4 & 132 & 42 & 7 & 0.138 & 0.155 & 0.87 & $0.58,1.30$ & 0.488 \\
\hline rs3735590 & $\mathrm{G} / \mathrm{A}$ & 161 & 16 & 0 & 156 & 25 & 0 & 0.045 & 0.069 & 0.60 & $0.31,1.18$ & 0.138 \\
\hline rs854550 & $\mathrm{G} / \mathrm{A}$ & 133 & 40 & 44 & 133 & 41 & 7 & 0.136 & 0.152 & 0.86 & $0.57,1.29$ & 0.472 \\
\hline
\end{tabular}

MAF, minor allele frequency; allele $1 / 2$, major/minor allele.

${ }^{*}$ Adjusted for sex and age.

†From the logistic regression analysis. 
their high LD $\left(r^{2} 1\right)$ value. These two SNP correspond to block 2.

SNP located in the PON1 3'UTR (rs854550, rs854551 and rs854552) comprise block 1 , and they were highly associated with paraoxonase activity and weakly associated with lactonase, arylesterase and diazoxonase activities. The intronic SNP rs3917527 was only associated with paraoxonase activity, and it was also excluded from the LD blocks.

\section{Discussion}

Many previous studies have investigated the association between PON1 genetic variations and the risk for certain diseases or changes in PON1 activities in the context of certain diseases; however, no study has considered both variables simultaneously. To date, this is the first study to demonstrate genetic associations between PON1 polymorphisms and prepubertal childhood obesity. Furthermore, the present study has used both intronic and exonic polymorphisms and four different substrates to measure serum PON1 activities. An association between obesity and the PON1 SNP rs854566 has not been reported previously, and we found that it was negatively associated with childhood obesity and also affected all four of the measurements of PON1 activities. Children carrying the minor allele of SNP rs854566 had higher diazoxonase, lactonase and arylesterase activities and lower paraoxonase activity. In contrast, the genetic variants Q192R and L55M were not significantly associated with childhood obesity, although they did exert effects on PON1 activities. To the best of our knowledge, three previous studies have tested for genetic associations between the PON1 gene and obesity. One study conducted on adolescents has reported no association between obesity and the Q192R polymorphism ${ }^{(18)}$. Another study conducted on obese Portuguese women has shown a positive association between the Q192R genotype and disease risk ${ }^{(17)}$. Lastly, a recent study conducted on obese Mexican adults has identified a positive association between the 55LL genotype and obesity ${ }^{(19)}$. However, these findings are inconsistent with those of the present study. It is likely that the complex nature of obesity, which depends on both genetic and environmental factors, is the cause for the unclear relationship between PON1 genetic variations, PON1 activities and childhood obesity. The importance of conducting the present study in prepubertal children should be noted as this population should have lower exposure levels to toxins (e.g. tobacco, alcohol and drugs) than adults and is much less likely to have chronic diseases that might affect PON1 activities.

PON1 activities were not found to be significantly altered in the obese group based on any of the metrics that we used. One study has reported lowered paraoxonase activity in obese adults ${ }^{(6)}$, whereas no changes in paraoxonase and arylesterase activities have been observed in another study ${ }^{(9)}$. The previous study that was conducted on children reported only measured paraoxonase and arylesterase activities, which were observed to be lower in fifty-nine obese children (11.95 (SEм 1.61) years) than in fifty-one normal-weight children (12.00 (sem 3.91) years) ${ }^{(8)}$. However, the fact that this 


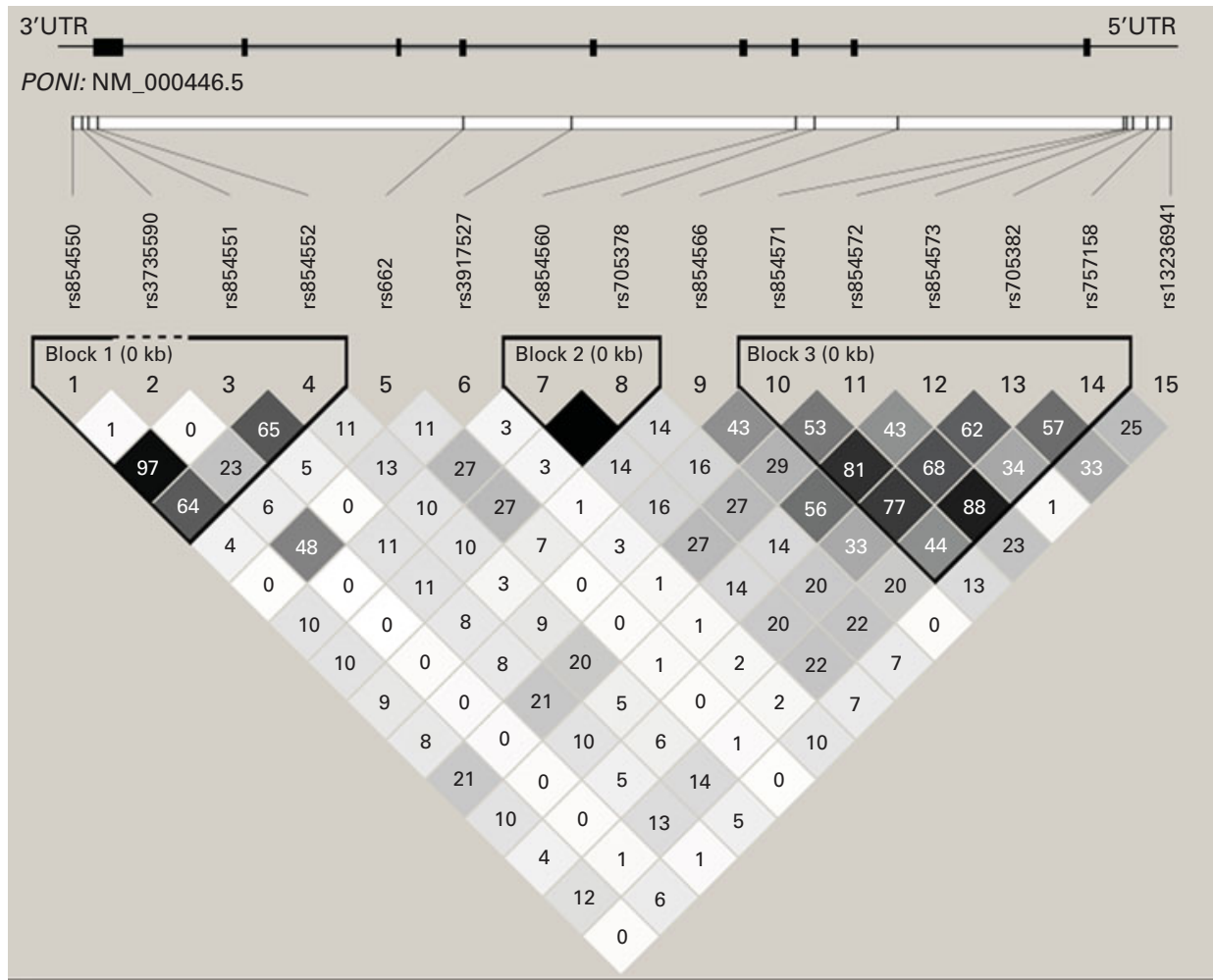

Fig. 1. Linkage disequilibrium diagram of the studied variants with the exon-intron scheme of the PON1 gene. The $r^{2}$ values between the genotyped SNP are shown in each cell; black cells correspond to $r^{2} 1.00$. Block definition followed the four-gamete rule using the Haploview software. (A colour version of this figure can be found online at http://www.journals.cambridge.org/bjn)

population was not defined as entirely prepubertal, as well as the relatively low sample size, could explain the divergent results. Furthermore, the fact that lactonase activity had never been measured in obese children is significant and should be addressed.

Interestingly, we determined a negative correlation between lactonase activity and the level of MPO, which is considered to be a reliable biomarker for endothelial dysfunction and CVD risk $^{(4)}$. Moreover, we observed a negative correlation between lactonase activity and the level of FAPB-4, a biomarker that has been linked to insulin resistance, the metabolic syndrome, hypertriacylglycerolaemia and atherosclerosis ${ }^{(26)}$. Therefore, the lack of any relationship between paraoxonase activity and the levels of these biomarkers supports the hypothesis that measuring other PON1 activities, rather than the paraoxonase activity, is more physiologically relevant for determining PON1 function ${ }^{(12)}$. Similarly, lactonase, arylesterase and diazoxonase activities were correlated with HDL-C, total cholesterol and ApoA1 levels, whereas paraoxonase activity was not. In this line, a recent study ${ }^{(27)}$ has shown that arylesterase activity is a more powerful indicator of cardiovascular risk than paraoxonase activity. Considering the present results and the previously published evidence, we propose that lactonase activity is a good indicator of PON1 function and that it should be used in further studies investigating the role of PON1 in the development of obesity and CVD.

Although the protective effects of SNP rs854566 on childhood obesity need to be validated, it should be noted that this allele was associated with increased lactonase activity, which itself was positively correlated with HDL-C, ApoA1, retinol and $\alpha$-tocopherol levels and negatively correlated with fatty acid-binding protein 4 and MPO levels. These relationships support a protective role for SNP rs854566 by linking the antioxidant and anti-inflammatory functions of PON1 with protection from the development of obesity and CVD. Indeed, it is known that HDL has anti-inflammatory and antioxidant functions in vivo, and it is believed from previous studies carried out in animal models that PON1 contributes to these functions ${ }^{(2,28)}$. However, although lactonase activity is most probably responsible for the antioxidant effects of PON1 ${ }^{(10,11,29)}$, we did not observe significant correlations with ox-LDL levels. The present findings are consistent with those of Carlson et $a l^{(30)}$, who reported no association between ox-LDL levels and arylesterase activity.

PON1 is expressed in the interstitial space of adipose tissue $^{(3)}$. Mature adipocytes have been reported to express cluster of differentiation 36 (CD36), which can recognise and bind to ox-LDL; these molecules are then endocytosed ${ }^{(31)}$ and can modulate adipose tissue mass by stimulating adipocyte proliferation and differentiation ${ }^{(32)}$. However, the function of PON1 in adipose tissue is unknown. Although we did not identify any relationship between PON1 activities and ox-LDL levels in the plasma, more studies should be performed to describe the effect of PON1 on ox-LDL levels in adipose tissue and to determine whether PON1 plays a role in adipocyte differentiation and proliferation during the development of obesity.

Several limitations in the present study should be noted. The negative association between the PON1 SNP rs854566 
and childhood obesity identified in the present study requires further validation in a larger population. Another potential limitation is that the measurements were only carried out under fasting conditions at a single time point. We, therefore, were unable to determine the variability and prognostic value of changing levels over time and of the impact of dietary or therapeutic interventions on serum PON1 activities.

\section{Conclusion}

The present results suggest a protective role for SNP rs854566 with respect to obesity. This SNP also exhibits strong associations with serum PON1 activities, namely increased diazoxonase, lactonase and arylesterase activities. Despite these results, serum PON1 activities were not found to be significantly different in prepubertal obese children. Nevertheless, we demonstrate that lactonase activity is a reliable indicator of PON1 function and should be used in future studies investigating the role of PON1 in the development of obesity and CVD. Future in vivo and in vitro studies on the function of PON1 in adipose tissue may help us to identify potential antioxidant and protective roles for PON1 in the protection against the development of obesity and its co-morbidities.

\section{Supplementary material}

To view supplementary material for this article, please visit http://dx.doi.org/10.1017/S0007114513001967

\section{Acknowledgements}

The authors thank the children and parents who participated in the study. The present study was funded by the Plan Nacional de Investigación Científica, Desarrollo e Innovación Tecnológica (I + D + I), the Instituto de Salud Carlos III-Fondo de Investigación Sanitaria (FIS) (PI 020826, PI051968), Redes temáticas de investigación cooperativa (RETIC) (Red SAMID RD08/0072/0028), Junta de Andalucía, the Consejería de Innovación y Ciencia (P06-CTS 2203), the Consejería de Salud (0098/2005) and the Ministerio de Ciencia e Innovación, Campus de Excelencia Internacional de Granada. GREIBCTS461. The authors' contributions were as follows: F. G., A. G. and C. M. A. conceptualised and designed the study; M. G.-C., R. L., R. T. and R. C. were involved in the data and sample collection; J. O. carried out the biomarker analysis; A. I. R. and O. L.-G. conducted the enzymatic analysis; A. I. R. performed the statistical analysis; A. I. R. and C. M. A. wrote the manuscript.

The authors declare no conflicts of interest that could be perceived as affecting the impartiality of this research.

\section{References}

1. Mackness MI, Arrol S, Abbot C, et al. (1993) Protection of low-density lipoprotein against oxidative modification by high-density lipoprotein associated paraoxonase. Atherosclerosis 104, 129-135.
2. Mackness MI, Durrington PN \& Mackness B (2000) How high-density lipoprotein protects against the effects of lipid peroxidation. Curr Opin Lipidol 11, 383-388.

3. Marsillach J, Parra S, Ferré N, et al. (2008) Paraoxonase-1 in chronic liver diseases, neurological diseases, and HIV infection. In The Paraoxonases: Their Role in Disease Development and Xenobiotic Metabolism, pp. 187-198 [B Mackness, M Mackness, M Aviram and G Paragh, editors]. Dordrecht: Springer.

4. Olza J, Aguilera CM, Gil-Campos M, et al. (2012) Myeloperoxidase is an early biomarker of inflammation and cardiovascular risk in prepubertal obese children. Diabetes Care $\mathbf{3 5}$, 2373-2376.

5. Marsillach J, Mackness B, Mackness M, et al. (2008) Immunohistochemical analysis of paraoxonases-1,2 and 3 expression in normal mouse tissues. Free Radic Biol Med 45, 146-157.

6. Aslan M, Horoz M, Sabuncu T, et al. (2011) Serum paraoxonase enzyme activity and oxidative stress in obese subjects. Pol Arch Med Wewn 121, 181-186.

7. Ferretti G, Bacchetti T, Moroni C, et al. (2005) Paraoxonase activity in high-density lipoproteins: a comparison between healthy and obese females. J Clin Endocrinol Metab 90, $1728-1733$.

8. Koncsos P, Seres I, Harangi M, et al. (2010) Human paraoxonase-1 activity in childhood obesity and its relation to leptin and adiponectin levels. Pediatr Res 67, 309-313.

9. Tabur S, Torun AN, Sabuncu T, et al. (2010) Non-diabetic metabolic syndrome and obesity do not affect serum paraoxonase and arylesterase activities but do affect oxidative stress and inflammation. Eur J Endocrinol 162, 535-541.

10. Rosenblat M, Gaidukov L, Khersonsky O, et al. (2006) The catalytic histidine dyad of high density lipoprotein-associated serum paraoxonase-1 (PON1) is essential for PON1mediated inhibition of low density lipoprotein oxidation and stimulation of macrophage cholesterol efflux. $J$ Biol Chem 281, 7657-7665.

11. Jakubowski H (2000) Calcium-dependent human serum homocysteine thiolactone hydrolase. A protective mechanism against protein N-homocysteinylation. J Biol Chem 275, 3957-3962.

12. Camps J, Marsillach J \& Joven J (2009) The paraoxonases: role in human diseases and methodological difficulties in measurement. Crit Rev Clin Lab Sci 46, 83-106.

13. Camps J, Mackness M, Mackness B, et al. (2010) Serum paraoxonase- 1 activity and genetic polymorphisms: common errors in measurement and interpretation of results. Clin Chem Lab Med 48, 893-894.

14. Costa LG, Giordano G \& Furlong CE (2011) Research update: pharmacological and dietary modulators of paraoxonase 1 (PON1) activity and expression: the hunt goes on. Biochem Pharmacol 81, 337-344.

15. Richter RJ \& Furlong CE (1999) Determination of paraoxonase (PON1) status requires more than genotyping. Pharmacogenetics 9, 745-753.

16. Furlong CE, Richter RJ, Li WF, et al. (2008) The functional consequences of polymorphisms in the human PON1 gene. In The Paraoxonases: Their Role in Disease Development and Xenobiotic Metabolism, pp. 267-281 [B Mackness, M Mackness, M Aviram and G Paragh, editors]. Dordrecht: Springer.

17. Veiga L, Silva-Nunes J, Melao A, et al. (2011) Q192R polymorphism of the paraoxonase-1 gene as a risk factor for obesity in Portuguese women. Eur J Endocrinol 164, 213-218.

18. Baráth A, Németh I, Karg E, et al. (2006) Roles of paraoxonase and oxidative stress in adolescents with uraemic, essential or 
obesity-induced hypertension. Kidney Blood Press Res 29, 144-151.

19. Martínez-Salazar MF, Almenares-López D, García-Jiménez S, et al. (2011) Relationship between the paraoxonase (PON1) L55M and Q192R polymorphisms and obesity in a Mexican population: a pilot study. Genes Nutr 6, 361-368.

20. Cole TJ, Bellizzi MC, Flegal KM, et al. (2000) Establishing a standard definition for child overweight and obesity worldwide: international survey. BMJ 320, 1240-1243.

21. Olza J, Gil-Campos M, Leis R, et al. (2012) A gene variant of $11 \beta$-hydroxysteroid dehydrogenase type 1 is associated with obesity in children. Int J Obes 36, 1558-1563.

22. Garcia-Rodriguez CE, Mesa MD, Olza J, et al. (2012) Does consumption of two portions of salmon per week enhance the antioxidant defense system in pregnant women? Antioxid Redox Signal 16, 1401-1406.

23. Purcell S, Neale B, Todd-Brown K, et al. (2007) PLINK: a toolset for whole-genome association and populationbased linkage analysis. Am J Hum Genet 81, 559-575.

24. Barrett JC, Fry B, Maller J, et al. (2005) Haploview: analysis and visualization of LD and haplotype maps. Bioinformatics 21, 263-265.

25. Hernández AF, Gil F, Leno $\mathrm{E}$, et al. (2009) Interaction between human serum esterases and environmental metal compounds. Neurotoxicology 30, 628-635.
26. Kralisch S \& Fasshauer M (2012) Adipocyte fatty acid binding protein: a novel adipokine involved in the pathogenesis of metabolic and vascular disease? Diabetologia 56, 10-21.

27. Tang WH, Hartiala J, Fan Y, et al. (2012) Clinical and genetic association of serum paraoxonase and arylesterase activities with cardiovascular risk. Arterioscler Thromb Vasc Biol 32, $2803-2812$

28. Mackness B, Quarck R, Verreth W, et al. (2006) Human paraoxonase-1 overexpression inhibits atherosclerosis in a mouse model of metabolic syndrome. Arterioscler Thromb Vasc Biol 26, 1545-1550.

29. Gaidukov L \& Tawfik DS (2007) The development of human sera tests for HDL-bound serum PON1 and its lipolactonase activity. J Lipid Res 48, 1637-1646.

30. Carlson CS, Heagerty PJ, Hatsukami TS, et al. (2006) TagSNP analyses of the $P O N$ gene cluster: effects on PON1 activity, LDL oxidative susceptibility, and vascular disease. J Lipid Res 47, 1014-1024.

31. Kuniyasu A, Hayashi S \& Nakayama H (2002) Adipocytes recognize and degrade oxidized low density lipoprotein through CD36. Biochem Biophys Res Commun 295 , 319-323.

32. Masella R, Vari R, D'Archivio M, et al. (2006) Oxidised LDL modulate adipogenesis in 3T3-L1 preadipocytes by affecting the balance between cell proliferation and differentiation. FEBS Lett 580, 2421-2429. 